\title{
Options Pricing in Jump Diffusion Markets during Financial Crisis
}

\author{
Youssef El-Khatib*, Mohamed Ali Hajji and Mohammed Al-Refai \\ Department of Mathematical Sciences, UAE University, 15551, Al-Ain, United Arab Emirates
}

Received: 25 Feb. 2013, Revised: 23 Jun. 2013, Accepted: 24 Jun. 2013

Published online: 1 Nov. 2013

\begin{abstract}
In this paper, we suggest a jump diffusion model in markets during financial crisis. Using risk-neutral pricing, we derive a partial differential equation (P.D.E.) for the prices of European options. We find a closed form solution of the P.D.E. in the particular case where the stock price is too large. Then, we use such a solution as a boundary condition in the numerical treatment of the P.D.E. for any range of stock price. The numerical method adopted is the unconditionally stable Crank-Nicolson method. Illustrative examples are presented.
\end{abstract}

Keywords: Jump-diffusion models, financial crisis, incomplete markets, European options, finite differences method, series solutions.

\section{Introduction}

Most of the works on modeling financial derivatives assume the continuity of the trajectories of the underlying asset prices. For instance, in the pioneer work of Black and Scholes [2] financial asset prices are modeled by the Brownian motion. One of the shortcomings of this model is that it doesn't consider the jumps which can occur in the prices randomly at any time. Indeed, many researchers studied models with jumps, see for example the model in [19] or more recently [6]. However, to the authors knowledge, there are no studies that investigate this issue during financial crises where the volatility is naturally higher than in normal situations.

Options pricing models coming from empirical studies of the dynamics of financial markets after the occurrence of a financial crash do not match with the stochastic models used in the literature. For instance, the work of [23] empirically shows that the post-crash dynamics follow a converging oscillatory motion. On the other hand, the paper of [17] shows that financial markets follow power-law relaxation decay. Several ideas have been suggested to overcome this shortcoming of the Black-Scholes model. In fact, new option pricing models have been developed based on empirical observations (see for instance [5], [21], [24], [7] and [18]). For example, in [9], the authors investigate the calibration properties of several multi-factor stochastic volatility models using a data set of vanilla options. In [8], the authors suggest a newer model which extends the Black-Scholes model. The extension takes into accounts the post-crash dynamics as proposed by [23]. The authors derive the following stochastic differential equation that couples the post-crash market index to individual stock prices,

$$
\frac{d S_{t}}{S_{t}}=\left(\mu+\frac{\mu_{1} g(t)}{S_{t}}\right) d t+\left(\sigma+\frac{\gamma g(t)}{S_{t}}\right) d W_{t},
$$

where $t \in[0, T]$ and $S_{0}=x>0$. The stochastic process $\left(W_{t}\right)_{t \in[0, T]}$ denotes a standard Brownian motion and $g(t)=$ $A+B e^{v t} \sin (\omega t)$, with $\mu, \mu_{1}, \sigma, \gamma, A, B, v$, and $\omega$ are real constants. The volatility of the original asset is denoted by $\sigma$. The authors obtained the following partial differential equation (P.D.E.) for the option price

$$
\frac{\partial C}{\partial t}+r S \frac{\partial C}{\partial S}-r C+\frac{1}{2}(\sigma S+\gamma g(t))^{2} \frac{\partial^{2} C}{\partial S^{2}}=0
$$

with the terminal condition $C(S, T)=(S-K)^{+}$, where $C$ is the call option's price, $r$ is the risk free rate, and $K$ is the strike price. They solved the P.D.E. using finite differences method. Also, the price sensitivities for model (1) have been calculated in [11].

In this this paper we extend the work of [8] to jump diffusion models. To the authors' best knowledge, this paper is the first attempt to suggest a model with jumps during financial crisis. In this work, we assume that the

\footnotetext{
*Corresponding author e-mail: Youssef_Elkhatib@uaeu.ac.ae
} 
dynamic of the underlying asset price is given by the stochastic differential equation

$$
d S_{t}=\left(\mu S_{t}+\mu_{1} g_{t}\right) d t+\left(\sigma S_{t}+\gamma g_{t}\right) d W_{t}+b \sigma S_{t} d M_{t},
$$

where $t \in[0, T]$ and $S_{0}=x>0$. The processes $\left(W_{t}\right)_{t \in[0, T]}$ and $\left(M_{t}\right)_{t \in[0, T]}$ denote respectively a standard Brownian motion and a compensated Poisson process. $\mu, \mu_{1}, b, \sigma, \gamma$ are constants, with $1+b \sigma>0$ and $g$ is a deterministic function. The contributions of this work are twofold. The first is the derivation of a P.D.E. for the price of European options. The second is the design of an unconditionally stable numerical scheme based on finite differences for the numerical solution of the problem. It is worth mentioning that for model (2) with no crisis $\left(\gamma g_{t}=0\right)$ a closed form solution has been obtained in [10] and the price sensitivities have been calculated using the Malliavin calculus in [12].

The rest of the paper is organized as follows. In Section 2, we introduce the jump diffusion model during financial crisis and we derive a P.D.E for European options price. In Section 3 we derive a closed form solution for the P.D.E. when the strike price is large. In Section 4, we use the solution obtained in Section 3 as a boundary solution to solve numerically the P.D.E. over the whole range of strike price, using an unconditionally stable numerical scheme based on Crank Nicolson dicretization. Illustrative example is presented. Section 5 concludes the paper with some remarks.

\section{Pricing European options in jump diffusion markets during financial crisis}

In this section, we introduce jump diffusion model during financial crisis and derive a governing P.D.E. for European options price. We need the following notations and terminologies. Let $\left(N_{t}\right)_{t \in[0, T]}$ be a Poisson process with deterministic intensity $\lambda$. Let $M_{t}=N_{t}-\lambda t$ be its associated compensated process and $\left(B_{t}\right)_{t \in[0, T]}$ be a Brownian motion. We assume that we work on a probability space $(\Omega, \mathscr{F}, P)$ with $\left(M_{t}\right)_{t \in[0, T]}$ and $\left(B_{t}\right)_{t \in[0, T]}$ independent. We denote by $\left(\mathscr{F}_{t}\right)_{t \in[0, T]}$ the filtration generated by $\left(N_{t}\right)_{t \in[0, T]}$ and $\left(B_{t}\right)_{t \in[0, T]}$.

\subsection{The model}

We consider a market with two assets: a risky asset related to a European call option and a riskless one. The maturity is $T$, the strike is $K$ and the payoff is $h\left(S_{T}\right)=\left(S_{T}-K\right)^{+} \equiv$ $\max \left\{S_{T}-K, 0\right\}$. The price of the riskless asset is given by

$$
d A_{t}=r A_{t} d t, \quad t \in[0, T], \quad A_{0}=1,
$$

where $r>0$ denotes the interest rate. The risky asset has a price $\left(S_{t}\right)_{t \in[0, T]}$ described by the stochastic differential equation

$$
d S_{t}=\left(\mu S_{t}+\mu_{1} g_{t}\right) d t+\left(\sigma S_{t}+\gamma g_{t}\right) d W_{t}+b \sigma S_{t} d M_{t},
$$

where $t \in[0, T]$ and $S_{0}=x>0$ and $\mu, \mu_{1}, b, \sigma, \gamma$ are constants, with $1+b \sigma>0$ and $g$ is a deterministic function. $\left(W_{t}\right)_{t \in[0, T]}$ and $\left(M_{t}\right)_{t \in[0, T]}$ are respectively the Brownian motion and the compensated Poisson process.

Remark. The parameter $b$ in the previous equation will determine the direction of the jumps ${ }^{1}$. In fact:

-If $b<0$ the jumps are pushing the stock down, the stock price is then decreasing at each jump.

-If $b=0$, there are no jumps.

-If $b>0$, the jumps are pushing the stock up, the stock price is then increasing at each jump.

\subsection{Change of probability}

As in the original work of [2], we assume that there is no place to arbitrage opportunity (in other words, no riskless profit). It is well known that there is no arbitrage if and only if there exists at least one Equivalent Martingale Measure (E.M.M.) (see the First Fundamental Theorem of Asset Pricing, [13] and [14]). Notice that a contingent claim is a random variable $H$ that represents the payoff at time $T$ from a seller to a buyer. For example, in our model with European call option, the payoff is $H=h\left(S_{T}\right)=\left(S_{T}-K\right)^{+}$.

By definition, a market is said to be complete if every contingent claim in the market is attainable, i.e., we can find a self-financing strategy whose value at maturity is equal to the claim's value. Recall that an arbitrage-free market is complete if and only if there is a unique E.M.M. (Second Fundamental Theorem of Asset Pricing, [13] and [14]). The market in our model is incomplete since there are infinitely many E.M.M. To see this, we first characterize the set of E.M.M. By definition a probability $Q$ is said to be a $P$-E.M.M. if it is equivalent to the historical probability $P$ and it satisfies the fact that the discounted prices are $Q$-martingales.

It is known that a probability $Q$ equivalent to $P$ is specified by its Radon-Nikodym density with respect to $P$ which can be expressed as

$$
\begin{aligned}
\rho_{T}= & \exp \left(\int_{0}^{T} \theta_{1 s} d W_{s}-\frac{1}{2} \int_{0}^{T} \theta_{1_{s}^{2}}^{2} d s\right) \exp \left(\int_{0}^{T} \ln (1\right. \\
& \left.\left.+\theta_{2 s}\right) d M_{s}+\int_{0}^{T} \lambda_{s}\left[\ln \left(1+\theta_{2 s}\right)-\theta_{2 s}\right] d s\right)
\end{aligned}
$$

where $\left(\theta_{1 t}\right)_{t \in[0, T]}$ and $\left(\theta_{2 t}\right)_{t \in[0, T]}$ are two $\mathbb{R}$-valued predictable processes with $\theta_{2}>-1$. Recall that $Q$ is a $P$-E.M.M. if the discounted prices $\left(\frac{S_{t}}{A_{t}}\right)_{t \in[0, T]}$ and $\left(\frac{A_{t}}{A_{t}}\right)_{t \in[0, T]}$ are $Q$-martingales which leads to a relation between $\theta_{1}$ and $\theta_{2}$ as formally stated in the following proposition.

\footnotetext{
$1 b$ affects also the jumps sizes.
} 
Proposition 1.Let $Q$ be a P-E.M.M. defined by its Radon-Nikodym density with respect to $P$ given in (3). Then $\left(\theta_{1 t}\right)_{t \in[0, T]}$ and $\left(\theta_{2 t}\right)_{t \in[0, T]}$ satisfy

$$
\mu S_{t}+\mu_{1} g_{t}-r S_{t}+\left(\sigma S_{t}+\gamma g_{t}\right) \theta_{1 t}+\lambda b \sigma S_{t} \theta_{2 t}=0 \text {. }
$$

Proof. $Q$ is a $P$-E.M.M. if the discounted prices are $Q$-martingales, which means that $\left(\frac{S_{t}}{A_{t}}\right)_{t \in[0, T]}$ is a $Q$-martingale which in turn means that $\left(\frac{S_{t}}{A_{t}} \rho_{t}\right)_{t \in[0, T]}$ is a $P$-martingale. The integration by parts formula (see [20]) gives

$$
\begin{aligned}
d\left(\frac{S_{t}}{A_{t}} \rho_{t}\right)= & d\left(e^{-r t} S_{t} \rho_{t}\right) \\
= & \rho_{t} d\left(e^{-r t} S_{t}\right)+e^{-r t} S_{t} d \rho_{t}+d\left[e^{-r t} S_{t}, \rho_{t}\right] \\
= & \rho_{t} d\left(e^{-r t} S_{t}\right)+e^{-r t} S_{t} d \rho_{t}+e^{-r t} d\left[S_{t}, \rho_{t}\right] \\
= & \rho_{t} e^{-r t}\left(d S_{t}-r S_{t} d t\right)+e^{-r t} S_{t} \rho_{t}\left(\theta_{1} d W_{t}\right. \\
& \left.+\theta_{2 t} d M_{t}\right)+e^{-r t} d\left[S_{t}, \rho_{t}\right]
\end{aligned}
$$

with

$$
\begin{aligned}
d\left[S_{t}, \rho_{t}\right]= & {\left[\left(\mu S_{t}+\mu_{1} g_{t}\right) d t+\left(\sigma S_{t}+\gamma g_{t}\right) d W_{t}+b \sigma S_{t} d M_{t}\right.} \\
& \left.\rho_{t}\left(\theta_{1 t} d W_{t}+\theta_{2 t} d M_{t}\right)\right] \\
= & {\left[\left(\sigma S_{t}+\gamma g_{t}\right) d W_{t}, \theta_{1 t} \rho_{t} d W_{t}\right] } \\
& +\left[b \sigma S_{t} d M_{t}, \theta_{2 t} \rho_{t} d M_{t}\right] \\
= & \left(\sigma S_{t}+\gamma g_{t}\right) \theta_{1 t} \rho_{t} d t+\left[b \sigma S_{t} d N_{t}, \theta_{2 t} \rho_{t} d N_{t}\right] \\
= & \left(\sigma S_{t}+\gamma g_{t}\right) \theta_{1 t} \rho_{t} d t+b \sigma S_{t} \theta_{2 t} \rho_{t} d N_{t} \\
= & \left(\left(\sigma S_{t}+\gamma g_{t}\right) \theta_{1 t} \rho_{t}+\lambda b \sigma S_{t} \theta_{2 t} \rho_{t}\right) d t \\
& +b \sigma S_{t} \theta_{2 t} \rho_{t} d M_{t}
\end{aligned}
$$

Therefore

$$
\begin{aligned}
d\left(\frac{S_{t}}{A_{t}} \rho_{t}\right)= & \left\{\left((\mu-r) S_{t}+\mu_{1} g_{t}\right) d t+\left(\sigma S_{t}+\gamma g_{t}\right) d W_{t}\right. \\
& +\left(b+\beta_{t}\right) \sigma S_{t} d M_{t}+S_{t}\left(\theta_{1 t} d W_{t}+\theta_{2 t} d M_{t}\right) \\
& \left.+\left(\left(\sigma S_{t}+\gamma g_{t}\right) \theta_{1 t}+\lambda b \sigma S_{t} \theta_{2 t}\right) d t\right\} \rho_{t} e^{-r t} \\
= & \left\{(\mu-r) S_{t}+\mu_{1} g_{t}+\left(\sigma S_{t}+\gamma g_{t}\right) \theta_{1 t}\right. \\
& \left.+\lambda b \sigma S_{t} \theta_{2 t}\right) d t+\left[\sigma S_{t}+\gamma g_{t}+S_{t} \alpha_{t}\right] d W_{t} \\
& \left.+\left[b \sigma S_{t}+S_{t} \theta_{2 t}+b \sigma S_{t} \theta_{2 t}\right] d M_{t}\right\} \rho_{t} e^{-r t}
\end{aligned}
$$

Since $\frac{S_{t}}{A_{t}} \rho_{t}$ is a $P$-E.M.M., it follows that

$$
\mu S_{t}+\mu_{1} g_{t}-r S_{t}+\left(\sigma S_{t}+\gamma g_{t}\right) \theta_{1 t}+\lambda b \sigma S_{t} \theta_{2 t}=0 .
$$

Equation (4) has infinitely many solutions in the set of predictable processes $\left(\theta_{1}, \theta_{2}\right)$ with $\theta_{2}>-1$. Moreover, each solution gives a $P$-E.M.M. $Q^{\theta_{1}, \theta_{2}}$. Consider the two processes $W^{\theta_{1}, \theta_{2}}$ and $M^{\theta_{1}, \theta_{2}}$ where

$$
\begin{aligned}
& W_{t}^{\theta_{1}, \theta_{2}}=W_{t}-\int_{0}^{t} \theta_{1 s} d s, \quad t \in[0, T], \\
& M_{t}^{\theta_{1}, \theta_{2}}=M_{t}-\int_{0}^{t} \lambda_{s} \theta_{2 s} d s, \quad t \in[0, T] .
\end{aligned}
$$

By Girsanov Theorem [15], $W^{\theta_{1}, \theta_{2}}$ is a $Q^{\theta_{1}, \theta_{2}}$-Brownian motion and $M^{\theta_{1}, \theta_{2}}$ is a $Q^{\theta_{1}, \theta_{2}}$-compensated Poisson process. From now on we work with the $P$-E.M.M. $\hat{Q}:=Q^{\hat{\theta}_{1}, 0}$ where we take $\theta_{2 t}=0^{2}$ and

$$
\hat{\theta_{1 t}}=\frac{(r-\mu) S_{t}-\mu_{1} g_{t}}{\sigma S_{t}+\gamma g_{t}} .
$$

Using equations (5)-(7), the underlying asset price can be written under $\hat{Q}$ as

$$
\begin{aligned}
d S_{t}= & \left(\mu S_{t}+\mu_{1} g_{t}\right) d t+\left(\sigma S_{t}+\gamma g_{t}\right) d W_{t}+b \sigma S_{t} d M_{t} \\
= & \left(\mu S_{t}+\mu_{1} g_{t}\right) d t+\left(\sigma S_{t}+\gamma g_{t}\right)\left[d \hat{W}_{t}+\hat{\theta}_{1 t} d t\right] \\
& +b \sigma S_{t} d M_{t} \\
= & \left(\mu S_{t}+\mu_{1} g_{t}\right) d t++b \sigma S_{t} d M_{t}+\left(\sigma S_{t}+\gamma g_{t}\right)\left[d \hat{W}_{t}\right. \\
& \left.+\frac{(r-\mu) S_{t}-\mu_{1} g_{t}}{\sigma S_{t}+\gamma g_{t}} d t\right] \\
= & \left(\mu S_{t}+\mu_{1} g_{t}+(r-\mu) S_{t}-\mu_{1} g_{t}\right) d t \\
& +\left(\sigma S_{t}+\gamma g_{t}\right) d \hat{W}_{t}+b \sigma S_{t} d M_{t} \\
= & r S_{t} d t+\left(\sigma S_{t}+\gamma g_{t}\right) d \hat{W}_{t}+b \sigma S_{t} d M_{t} .
\end{aligned}
$$

Figures 1 and 2 display two trajectories for the stock price at terminal time $T=1$ with a time step of 0.001 , for two different values of the volatility $\sigma=0.2$ and $\sigma=0.7$. The other parameter values used are: the initial stock price $S_{0}=7$, the jump intensity of the Poisson process $\lambda=3$, the interest rate $r=0.04$, the jump parameter $b=-1$, the crisis parameter $\gamma=0.02$, and the crisis function $g(t)=$ $e^{2 t} \sin (\pi t)$.

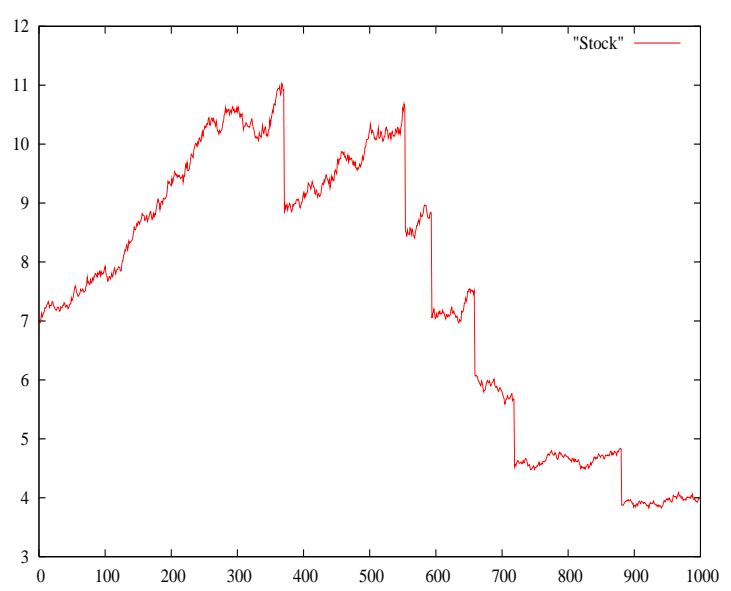

Fig. 1: A trajectory for the stock price when $\sigma=0.2$.

\footnotetext{
$2 \hat{Q}$ is exactly the $P$-E.M.M. that minimizes the entropy. The reader can found other types of risk measures such as the Coherent Risk Measure (C.R.M.) in [1] or more recently in [4].
} 


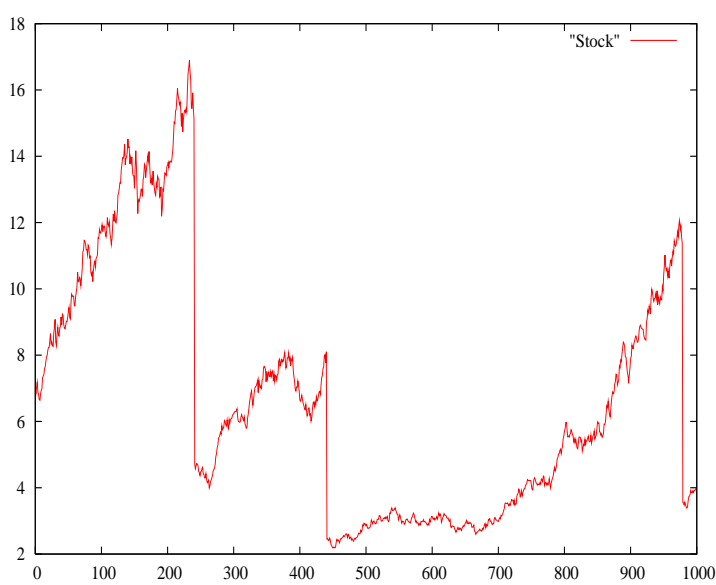

Fig. 2: A trajectory for the stock price when $\sigma=0.7$.

\subsection{P.D.E. of the option price}

Consider a European call option with underlying asset $\left(S_{t}\right)_{t \in[0, T]}$ given by equation (8) and a strike price $K$. By risk-neutral pricing, we can express the price of a European call option as a function $\hat{C}\left(S_{t}, t\right)$. In order to derive the P.D.E. for the European option price, we need Itô formula which is given by the following lemma (see [20]).

Lemma 1.Let $f, l$, and $k$ be three adapted processes such that

$\int_{0}^{t}\left|f_{s}\right| d s<\infty, \quad \int_{0}^{t}\left|l_{s}\right|^{2} d s<\infty, \quad$ and $\quad \int_{0}^{t} \lambda_{s}\left|k_{s}\right| d s<\infty$.

Let $X=\left(X_{t}\right)_{t \in[0, T]}$ be the process defined by

$$
d X_{t}=f_{t} d t+l_{t} d \hat{W}_{t}+k_{t} d M_{t} .
$$

For any function $F \in \mathscr{C}^{1,2}([0, T] \times \mathbb{R})$, we have

$$
\begin{aligned}
F\left(X_{t}, t\right)= & F\left(X_{0}, 0\right)+\int_{0}^{t}\left(\partial_{s} F\left(X_{s}, s\right)+\frac{1}{2} l_{s}^{2} \partial_{x x}^{2} F\left(X_{s^{-}}, s\right)\right. \\
& \left.+\left(f_{s}-k_{s} \lambda_{s}\right) \partial_{x} F\left(X_{s^{-}}, s\right)\right) d s \\
& +\int_{0}^{t} l_{s} \partial_{x} F\left(X_{s^{-}}, s\right) d \hat{W}_{s} \\
& +\sum_{s \leq t}\left(F\left(X_{s}, s\right)-F\left(X_{s^{-}}, s\right)\right)
\end{aligned}
$$

Equation (9) can be written in the following form

$$
\begin{aligned}
F\left(X_{t}, t\right)= & F\left(X_{0}, 0\right)+\int_{0}^{t}\left[\partial_{s} F\left(X_{s}, s\right)+\frac{1}{2} l_{s}^{2} \partial_{x x}^{2} F\left(X_{s^{-}}, s\right)\right. \\
& +\left(f_{s}-k_{s} \lambda_{s}\right) \partial_{x} F\left(X_{s^{-}}, s\right)+\lambda_{s}\left(F\left(X_{s^{-}}+k_{s}, s\right)\right. \\
& \left.\left.-F\left(X_{s^{-}}, s\right)\right)\right] d s+\int_{0}^{t} l_{s} \partial_{x} F\left(X_{s^{-}}, s\right) d \hat{W}_{s} \\
& +\int_{0}^{t}\left[F\left(X_{s^{-}}+k_{s}, s\right)-F\left(X_{s^{-}}, s\right)\right] d M_{s} .
\end{aligned}
$$

The following proposition gives the governing P.D.E. for European call option prices.

Proposition 2.The P.D.E. of the price of the European option in the jump diffusion model (8) is given by

$$
\begin{aligned}
& \partial_{t} \hat{C}(s, t)+(r s-b \sigma s \lambda) \partial_{x} \hat{C}(s, t)+\frac{1}{2}\left(\sigma s+\gamma g_{t}\right)^{2} \partial_{x x}^{2} \hat{C}(s, t) \\
& +\lambda(\hat{C}(s+b \sigma s, t)-\hat{C}(s, t))=r \hat{C}(s, t), \\
& \hat{C}(s, T)=(s-K)^{+} .
\end{aligned}
$$

Proof.Let $\hat{V}_{t}$ denote the value of the portfolio. Suppose that we are required to find a portfolio $\left(\hat{\zeta}_{t}, \hat{\eta}_{t}\right)_{t \in[0, T]}$ which leads to the payoff value $\hat{V}_{T}=h\left(S_{T}\right)=\hat{C}\left(S_{T}, T\right)$. We have for $t \in[0, T], \hat{V}_{t}=\hat{\zeta}_{t} A_{t}+\hat{\eta}_{t} S_{t}$. Since the strategy is assumed to be self-financing, we have, for $t \in[0, T]$,

$$
\begin{aligned}
d \hat{V}_{t}= & \hat{\zeta}_{t} d A_{t}+\hat{\eta}_{t} d S_{t} \\
= & \left(\hat{V}_{t}-\hat{\eta}_{t} S_{t}\right) A_{t}^{-1}\left(r A_{t} d t\right)+\hat{\eta}_{t}\left(r S_{t} d t+\left(\sigma S_{t}+\gamma g_{t}\right) d \hat{W}_{t}\right. \\
& \left.+b \sigma S_{t} d M_{t}\right) \\
= & r \hat{V}_{t} d t+\hat{\eta}_{t}\left(\sigma S_{t}+\gamma g_{t}\right) d \hat{W}_{t}+\hat{\eta}_{t} b \sigma S_{t} d M_{t} .
\end{aligned}
$$

By applying Itô formula (10) to $\hat{C}\left(S_{t}, t\right)$, we obtain, for any $t \in[0, T]$,

$$
\begin{aligned}
d \hat{C}\left(S_{t}, t\right)= & {\left[\partial_{t} \hat{C}\left(S_{t}, t\right)+\left(r S_{t}-b \sigma S_{t} \lambda\right) \partial_{x} \hat{C}\left(S_{t^{-}}, t\right)\right.} \\
& +\lambda\left(\hat{C}\left(S_{t^{-}}+b \sigma S_{t^{-}}, t\right)-\hat{C}\left(S_{t^{-}}, t\right)\right) \\
& \left.+\frac{1}{2}\left(\sigma S_{t}+\gamma g_{t}\right)^{2} \partial_{x x}^{2} \hat{C}\left(S_{t^{-}}, t\right)\right] d t \\
& +\left(\sigma S_{t}+\gamma g_{t}\right) \partial_{x} \hat{C}\left(S_{t^{-}}, t\right) d \hat{W}_{t} \\
& +\left[\hat{C}\left(S_{t^{-}}+b \sigma S_{t^{-}}, t\right)-\hat{C}\left(S_{t^{-}}, t\right)\right] d M_{t}
\end{aligned}
$$

If we seek a wealth $\hat{V}_{t}=\hat{C}\left(S_{t}, t\right)$, equating (13) and (14) yields

$$
\begin{aligned}
r_{t} \hat{C}\left(S_{t}, t\right)= & \partial_{t} \hat{C}\left(S_{t}, t\right)+\left(r S_{t}-b \sigma S_{t} \lambda\right) \partial_{x} \hat{C}\left(S_{t^{-}}, t\right) \\
& +\frac{1}{2}\left(\sigma S_{t}+\gamma g_{t}\right)^{2} \partial_{x x}^{2} \hat{C}\left(S_{t^{-}}, t\right) \\
& +\lambda\left(\hat{C}\left(S_{t^{-}}+b \sigma S_{t^{-}}, t\right)-\hat{C}\left(S_{t^{-}}, t\right)\right) \\
\hat{C}\left(S_{T}, T\right)= & \left(S_{T}-K\right)^{+} .
\end{aligned}
$$

which is the desired P.D.E. and condition, equations (11) and (12).

\section{A particular solution for the option price}

In this section we derive a boundary solution of the P.D.E. (11) subject to (12) for $s>K$. Precisely, we consider the P.D.E.

$$
\begin{aligned}
& C_{t}(s, t)+(r-\lambda \sigma b) s C_{s}(s, t)+\frac{1}{2}(\sigma s+\gamma g(t))^{2} C_{s s}(s, t) \\
& +\lambda C(s(1+\sigma b), t)-(\lambda+r) C(s, t)=0,
\end{aligned}
$$

for $s>K$ and $0 \leq t \leq T$, subject to the condition

$$
C(s, T)=s-K \equiv h(s), s>K .
$$


We should mention that this solution is very important as it will serve as a boundary solution for the numerical scheme (described next section) to solve (15) for $0 \leq s \leq$ $s_{\infty}$ for some bound $s_{\infty}$.

For ease of notation, let $\alpha_{1}=(r-\lambda \sigma b), \alpha_{2}(s, t)=\frac{1}{2}(\sigma s+\gamma g(t))^{2}, \alpha_{3}=\lambda$, $\alpha_{4}=(\lambda+r)$, and $\beta=1+\sigma b$. Then, integrating (15) with respect to $t$ from $t$ to $T$ and using (16),we obtain

$$
\begin{aligned}
C(s, t)= & h(s)+\int_{t}^{T}\left(\alpha_{1} s C_{s}(s, \tau)+\alpha_{2}(s, \tau) C_{s s}(s, \tau)\right. \\
& \left.+\alpha_{3} C(\beta s, \tau) d \tau-\alpha_{4} C(s, \tau)\right) d \tau
\end{aligned}
$$

Expand the solution formally as an infinite sum $C(s, t)=$ $\sum_{n=0}^{\infty} C_{n}(s, t)$. Substituting this expansion into the above, we obtain

$$
\begin{aligned}
\sum_{n=0}^{\infty} C_{n}(s, t)= & h(s)+\sum_{k=0}^{\infty} \int_{t}^{T}\left(\alpha_{1} s \partial_{s} C_{n}(s, \tau)+\alpha_{3} C_{n}(\beta s, \tau)\right. \\
& \left.+\alpha_{2}(s, \tau) \partial_{s}^{2} C_{n}(s, \tau)-\alpha_{4} C_{n}(s, \tau)\right) d \tau, \quad(17)
\end{aligned}
$$

One way to balance (17) is to let $C_{0}(s, t)=h(s)$ and obtain the following recursion relation for $C_{n}(s, t)$ :

$$
\begin{aligned}
C_{n+1}(s, t)= & \int_{t}^{T}\left(\alpha_{1} s \partial_{s} C_{n}(s, \tau)+\alpha_{2}(s, \tau) \partial_{s}^{2} C_{n}(s, \tau)\right. \\
& \left.+\alpha_{3} C_{n}(\beta s, \tau)-\alpha_{4} C_{n}(s, \tau)\right) d \tau .
\end{aligned}
$$

Since $\partial_{s}^{2} C_{0}(s, t)=0$, the above recursion relation reduces to

$$
\begin{aligned}
C_{n+1}(s, t)= & \int_{t}^{T}\left(\alpha_{1} s \partial_{s} C_{n}(s, \tau)+\alpha_{3} C_{n}(\beta s, \tau)\right. \\
& \left.-\alpha_{4} C_{n}(s, \tau)\right) d \tau, n \geq 0
\end{aligned}
$$

The following Lemma gives the solution of the recursion relation (19).

Lemma 2.The solution components $C_{n}(s, t), \quad n \geq 1$, satisfying (19) are given by

$$
C_{n}(s, t)=-K(-r)^{n} \frac{(T-t)^{n}}{n !}
$$

Proof. The proof is by induction. For $k=1$, from (19), we have

$$
C_{1}(s, t)=\int_{t}^{T}\left[\alpha_{1} s \partial_{s} C_{0}(s, \tau)+\alpha_{3} C_{0}(\beta s, \tau)-\alpha_{4} C_{0}(s, \tau)\right] d \tau .
$$

With $C_{0}(s, t)=s-K$, using the definitions of $\alpha_{1}, \alpha_{3}, \alpha_{4}$, and $\beta$, the integrand in (21) becomes

$$
\alpha_{1} s \partial_{s} C_{0}(s, \tau)+\alpha_{3} C_{0}(\beta s, \tau)-\alpha_{4} C_{0}(s, \tau)=r K .
$$

Thus, $c_{1}(s, t)=r(T-t) K$ which proves (20) for $n=1$.

Next, assume that (20) is true for $n=k$. From (19) and using the induction hypothesis (20), we obtain

$$
\begin{aligned}
C_{k+1}(s, t) & =\int_{t}^{T}-\left(\alpha_{3}-\alpha_{4}\right) K(-r)^{k} \frac{(T-\tau)^{k}}{k !} d \tau \\
& =-K(-r)^{k+1} \frac{(T-\tau)^{k+1}}{(k+1) !},
\end{aligned}
$$

which proves (20) for $n=k+1$. Hence (20) is true for all $n \geq 1$.

Based on Lemma 2, the solution of the P.D.E. (15) with (16) for $s>K$ is given in the following theorem.

Theorem 1.The solution of (15)-(16) for $s>K$ is given by

$$
C(s, t)=s-K e^{-r(T-t)} .
$$

Proof.By Lemma 2, we have $C_{n}(s, t)=-K(-r)^{n} \frac{(T-t)^{n}}{n !}, n \geq 1$. Then

$$
\begin{aligned}
C(s, t) & =\sum_{n=0}^{\infty} C_{n}(s, t)=C_{0}(s, t)+\sum_{n=1}^{\infty} C_{n}(s, t) \\
& =(s-K)+\sum_{n=1}^{\infty}\left(-K(-r)^{n} \frac{(T-t)^{n}}{n !}\right) \\
& =(s-K)-K\left(e^{-r(T-t)}-1\right)=s-K e^{-r(T-t),}
\end{aligned}
$$

which proves (22).

\section{Numerical computing of options prices}

In this section, we develop and implement a stable numerical scheme for the solution of (15) for $0 \leq s \leq s_{\infty}$ and $0 \leq t \leq T$. Similar treatments for different options price P.D.Es. or financial problems have been done in [3], [16] and [22]. We consider the following linear P.D.E.

$$
\frac{\partial C}{\partial t}+\alpha_{1} s \frac{\partial C}{\partial s}+\alpha_{2}(s, t) \frac{\partial^{2} C}{\partial s^{2}}+\alpha_{3} C(\beta s, t)-\alpha_{4} C=0
$$

with the initial and boundary conditions

$$
\begin{aligned}
& C(0, t)=0, \quad C(T, s)=H(s)=\max \{s-K, 0\}, \\
& C(s, t)=R(s, t), \quad \forall s \geq s_{\infty},
\end{aligned}
$$

where $R(s, t)=s-K e^{-r(T-t)}$, as derived in the previous section. The constants $\alpha_{0}, \alpha_{1}, \alpha_{3}, \alpha_{4}, \beta$, and $\alpha_{2}(s, t)$ are as defined in the previous section, namely,

$$
\alpha_{1}=(r-\lambda \sigma b), \alpha_{2}(s, t)=\frac{1}{2}(\sigma s+\gamma g(t))^{2}, \alpha_{3}=\lambda
$$

and

$$
\alpha_{4}=(\lambda+r), \beta=1+\sigma b .
$$

First, the problem is transformed into a "forward" initialvalue problem. Let $\tau=T-t$ and $\tilde{C}(s, \tau) \equiv C(s, t)$. Then equation (23) becomes

$$
-\frac{\partial \tilde{C}}{\partial \tau}+\alpha_{1} s \frac{\partial \tilde{C}}{\partial s}+\widetilde{\alpha}_{2}(s, \tau) \frac{\partial^{2} \tilde{C}}{\partial s^{2}}+\alpha_{3} \tilde{C}(\beta s, \tau)-\alpha_{4} \tilde{C}=0
$$

with the new conditions

$$
\begin{aligned}
& \tilde{C}(0, \tau)=0, \tilde{C}(s, 0)=H(s), \\
& \tilde{C}(s, \tau)=\tilde{R}(s, \tau)=R(s, T-\tau), \forall s \geq s_{\infty},
\end{aligned}
$$


and

$$
\widetilde{\alpha}_{2}(s, \tau)=\alpha_{2}(s, T-\tau) .
$$

Next, discretize the intervals $[0, T]$ and $\left[0, s_{\infty}\right]$ into equally spaced nodes:

$$
\begin{gathered}
\tau_{i}=i k, 0 \leq i \leq N_{t}, k=\frac{T}{N_{t}}, \\
s_{j}=j h, 0 \leq j \leq N_{s}, h=\frac{s_{\infty}}{N_{s}},
\end{gathered}
$$

where $k$ and $h$ are the step sizes. Let $\tilde{C}_{j}^{i} \approx \tilde{C}\left(s_{j}, \tau_{i}\right)$. Then using Crank Nicolson discetization, Equation becomes

$$
\frac{\tilde{C}_{j}^{i+1}-\tilde{C}_{j}^{i}}{k}=\frac{1}{2}\left(F_{j}^{i+1}+F_{j}^{i}\right)
$$

where

$$
\begin{aligned}
F_{j}^{i}= & \alpha_{1} s_{j} \frac{\tilde{C}_{j+1}^{i}-\tilde{C}_{j-1}^{i}}{2 h}+\widetilde{\alpha}_{2}\left(s_{j}, \tau_{i}\right) \frac{\tilde{C}_{j+1}^{i}-2 \tilde{C}_{j}^{i}+\tilde{C}_{j-1}^{i}}{h^{2}} \\
& +\alpha_{3} \tilde{C}\left(\beta s_{j}, \tau_{i}\right)-\alpha_{4} \tilde{C}_{j}^{i} .
\end{aligned}
$$

Equation (29) simplifies to

$$
\begin{aligned}
& a_{j}^{i+1} \tilde{C}_{j-1}^{i+1}+\left(1+b_{j}^{i+1}\right) \tilde{C}_{j}^{i+1}+c_{j}^{i+1} \tilde{C}_{j+1}^{i+1} \\
& -\frac{1}{2} k \alpha_{3} \tilde{C}\left(\beta s_{j}, \tau_{i+1}\right)=-a_{j}^{i} \tilde{C}_{j-1}^{i}+\left(1-b_{j}^{i}\right) \tilde{C}_{j}^{i}-c_{j}^{i} \tilde{C}_{j+1}^{i} \\
& +\frac{1}{2} k \alpha_{3} \tilde{C}\left(\beta s_{j}, \tau_{i}\right),
\end{aligned}
$$

where

$a_{j}^{i}=\frac{k}{4} j \alpha_{1}-\frac{k}{2 h^{2}} \widetilde{\alpha}_{j}^{i}, \quad b_{j}^{i}=\frac{k}{h^{2}} \widetilde{\alpha}_{j}^{i}+\frac{k}{2} \alpha_{4}$,

$c_{j}^{i}=-\frac{k}{4} j \alpha_{1}-\frac{k}{2 h^{2}} \widetilde{\alpha}_{j}^{i}$ and $\widetilde{\alpha}_{j}^{i}=\widetilde{\alpha}_{2}(j h, i k)$.

Note that from (27) and (28), we have

$$
\begin{aligned}
& \tilde{C}_{0}^{i}=0, \forall i \geq 1, \quad \tilde{C}_{j}^{0}=H(j h), \forall j \geq 1, \\
& \tilde{C}_{j}^{i}=\tilde{R}_{j}^{i} \equiv \tilde{R}\left(s_{j}, \tau_{i}\right), \forall j \geq N_{x} .
\end{aligned}
$$

In equation (30), the value of $\beta s_{j}$ in $\tilde{C}\left(\beta s_{j}, \tau_{m}\right)$ for $m=$ $i, i+1$, may be (i) outside the computational interval $\left[0, s_{\infty}\right]$ or (ii) inside the computational interval but not one of the mesh points $s_{j}$. To overcome this setback we suggest the following.

1.If $\beta s_{j} \geq s_{\infty}$, that is for $j \geq j^{*}=\left\lceil N_{x} / \beta\right\rceil$, then we let

$$
\tilde{C}\left(\beta s_{j}, \tau_{m}\right)=\tilde{R}\left(\beta s_{j}, \tau_{m}\right)=R\left(\beta s_{j}, T-\tau_{m}\right) .
$$

2.If $\beta s_{j}<s_{\infty}$, i.e., for $j<j^{*}$, let $n_{j}, 0 \leq n_{j} \leq N_{x}-1$, be the integer such that $s_{n_{j}} \leq \beta s_{j} \leq s_{n_{j}+1}$, i.e., $n_{j}=\lfloor\beta j\rfloor$. Then $\tilde{C}\left(\beta s_{j}, \tau_{m}\right)$ is approximated by the weighted average of $\tilde{C}_{n_{j}}^{m}$ and $\tilde{C}_{n_{j}+1}^{m}$ :

$$
\tilde{C}\left(\beta s_{j}, \tau_{m}\right) \approx w_{j} \tilde{C}_{n_{j}}^{m}+\left(1-w_{j}\right) \tilde{C}_{n_{j}+1}^{m}, w_{j}=\left(n_{j}-\beta j+1\right) .
$$

Note that in the case of $n_{j}=N_{x}-1$, we have $s_{N_{x}-1} \leq$ $\beta s_{j} \leq s_{\infty}$. In this case we let

$$
\tilde{C}\left(\beta s_{j}, \tau_{m}\right) \approx w_{j} \tilde{C}_{N_{x}-1}^{m}+\left(1-w_{j}\right) \tilde{R}\left(s_{\infty}, \tau_{m}\right)
$$

In summary, we have

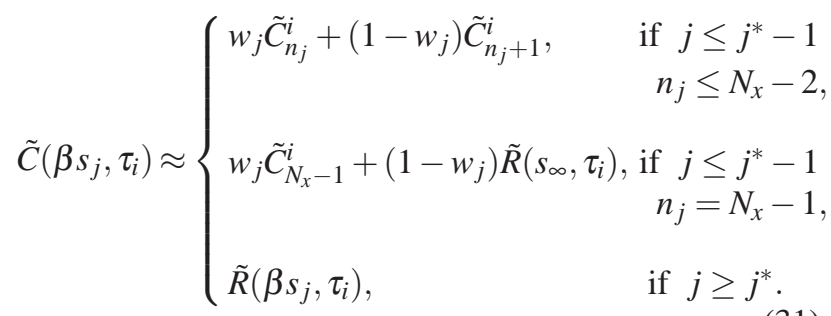

Now, let $C^{i}=\left[\begin{array}{llll}\tilde{C}_{1}^{i} & \tilde{C}_{2}^{i} \ldots \tilde{C}_{N_{x}-1}^{i}\end{array}\right]^{t}$. Using (31), equation (30) can be written in matrix form as

$$
\left(\mathbf{I}+\mathbf{A}^{i+1}\right) C^{i+1}-\mathbf{B} C^{i+1}-F^{i+1}=\left(\mathbf{I}-\mathbf{A}^{i}\right) C^{i}+\mathbf{B} C^{i}+F^{i},
$$

where $\mathbf{A}$ is an $\left(N_{x}-1\right) \times\left(N_{x}-1\right)$ tridiagonal matrix and $F^{i}$ is a column vector, given by

$$
\begin{aligned}
& \mathbf{A}^{i}=\left[\begin{array}{ccccc}
b_{1}^{i} & c_{1}^{i} & 0 & \ldots & 0 \\
a_{2}^{i} & b_{2}^{i} & c_{2}^{i} & \ldots & 0 \\
\vdots & \ddots & \ddots & \ddots & \vdots \\
\vdots & \ddots & a_{N_{x}-2}^{i} & b_{N_{x}-2}^{i} & c_{N_{x}-2}^{i} \\
0 & \ldots & \ldots & a_{N_{x}-1}^{i} & b_{N_{x}-1}^{i}
\end{array}\right], \\
& F_{j}^{i}= \begin{cases}\frac{1}{2} k \alpha_{3} \tilde{R}\left(\beta s_{j}, \tau_{i}\right), & j^{*} \leq j \leq N_{x}-2, \\
\frac{1}{2} k \alpha_{3} \tilde{R}\left(\beta s_{j}, \tau_{i}\right)-c_{N_{x}-1}^{i} \tilde{R}\left(s_{\infty}, \tau_{i}\right), & j=N_{x}-1, \\
\frac{1}{2} k \alpha_{3}\left(1-w_{j}\right) \tilde{R}\left(s_{\infty}, \tau_{i}\right), & j \leq j^{*}-1 \\
& n_{j}=N_{x}-1, \\
0, & \text { otherwise }\end{cases}
\end{aligned}
$$

and $\mathbf{B}$ is a sparse $\left(N_{x}-1\right) \times\left(N_{x}-1\right)$ matrix given by

$$
\begin{cases}\mathbf{B}_{j, i}=0, & \text { if } j \geq j^{*} \\ \mathbf{B}_{j, n_{j}}=\frac{1}{2} k \alpha_{3} w_{j}, & \text { if } j \leq j^{*}-1 \& n_{j} \leq N_{x}-1 \\ \mathbf{B}_{j, n_{j}+1}=\frac{1}{2} k \alpha_{3}\left(1-w_{j}\right) & \text { if } j \leq j^{*}-1 \& n_{j} \leq N_{x}-2\end{cases}
$$

The above outlined scheme has been implemented to obtain a numerical solution to system (32). Figures 3 and 4 depict the option prices for $\lambda=3, r=0.04, K=8, b=1$, $s_{\infty}=20, T=1, k=0.01 ; h=0.1, g(t)=e^{2 t} \sin (\pi t)$, and $\sigma=0.2$ (figure. 3) for $\sigma=0.7$ (figure. 4).

\section{Conclusion}

In this paper, we have considered a jump diffusion model during financial crisis. This new model takes into account two shortcomings of the standard Black-Scholes model, the jump and the increase in volatility during a financial crisis. The pricing option problem for the suggested model has been analyzed and a new partial differential equation for the option price has been derived. The derived P.D.E. reduces to the well-known Black-Scholes P.D.E. when the jump and the crisis parameters are not considered. 


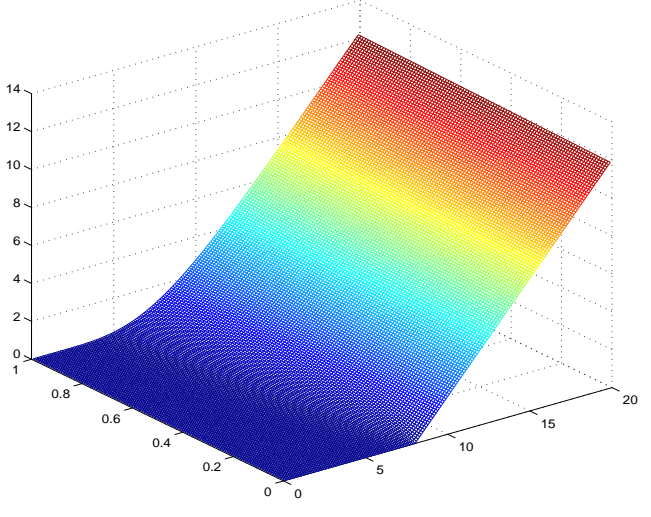

Fig. 3: An option price trajectory when $\sigma=0.2$.

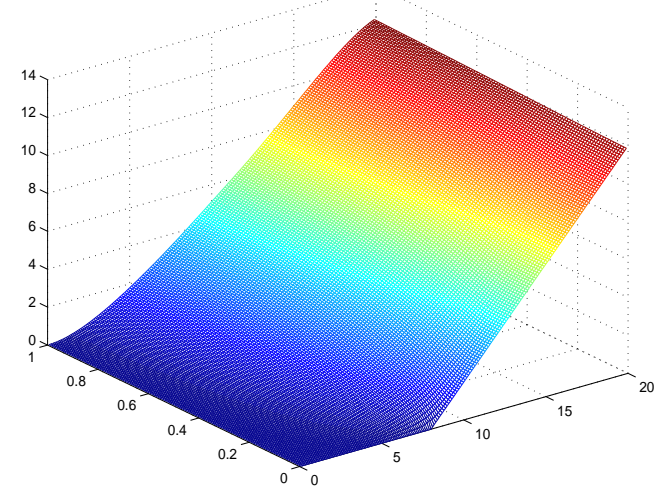

Fig. 4: An option price trajectory when $\sigma=0.7$.

For large values of the underlying asset price, we have derived a closed form solution of the new partial differential equation. This solution has been used as a boundary condition for the numerical treatment of the problem. A Crank-Nicolson based numerical scheme has been developed and implemented to obtain a numerical solution. The numerical results conform with the expected behavior of the option price.

\section{References}

[1] P. Artzner, F. Delbaen, J.-M. Eber, D. Heath, Thinking coherentlys, Risk, 10, 68-71 (1997).

[2] F. Black and M. Scholes, The Pricing of Options and Corporate Liabilities, Journal of Political Economy, 81, 637654 (1973).

[3] A. Aghajani, E. P. Kallehbasti, Common fixed point for generalized weak contractions in metric spaces and application to nonlinear integral equations, Mathematical Sciences Letters, 2, 87-97 (2013).

[4] Z. B. Walters, Quantum Coherent Dynamics at Ambient Temperature in Photosynthetic Molecules, Quantum Physics Letters, 1, 21-33 (2012).

[5] E. Deeba, G. Dibeh, and S. Xie, An algorithm for solving bond pricing problem, Appl. Math. Comput., 128, 81-94 (2002).

[6] L. Denis, C. Martini, A theoretical framework for the pricing of contingent claims in the presence of model uncertainty, Annals of Applied Probability, 16, 827-852 (2006).

[7] G. Dibeh and G. Chahda, Option pricing in markets with noisy cyclical and crash dynamics, Finance Lett., 3, 25-32 (2005).

[8] G. Dibeh and H.M. Harmanani, Option pricing during postcrash relaxation times, Physica A, 380, 357-365 (2007).

[9] J. Da Fonsecaa and M. Grassellibc, Riding on the smiles, Quantitative Finance, 11, 1609-1632 (2011).

[10] Y. El-Khatib, Q. M. Al-Mdallal, Numerical simulations for the pricing of options in jump diffusion markets, Arab Journal of Mathematical Sciences, 18, 199-208 (2012).

[11] Y. El-Khatib, A. Hatemi-J, Computations of Price Sensitivities after a Financial Market Crash, IAENG International Journal of Applied Mathematics, 41, 339-342 (2011).

[12] Y. El-Khatib, A. Hatemi-J, On the Calculation of Price Sensitivities with a Jump-Diffusion Structure, Journal of Statistics Applications \& Probability, 1, 171-182 (2012).

[13] J. M. Harrison and D. M. Kreps, Martingales and arbitrage in multiperiod securities markets, J. Econom. Theory, 20, 381-408 (1979).

[14] J. M. Harrison and S. R. Pliska, Martingales and stochastic integrals in the theory of continuous trading, Stochastic Processes and their Applications, 11, 215-260 (1981).

[15] J. Jacod, Calcul stochastique et problèmes de martingales, Lecture Notes in Mathematics, Springer Verlag, 714, (1979).

[16] A. Kiliçman and H. Eltayeb, Note on Partial Differential Equations with Non-Constant Coefficients and Convolution Method, Appl. Math. Inf. Sci., 6, 59-63 (2012).

[17] F. Lillo and F. Mantenga, Power-law relaxation in a complex system: Omori law after a financial market crash, Phys. Rev. E, 68, (2003).

[18] J. McCauley, The Dynamics of Markets: Econophysics and Finance, Cambridge University Press, Cambridge, (2004).

[19] R. C. Merton, Option pricing when underlying stock returns are discontinuous, Journal of Financial Economics, 3, 125144 (1976).

[20] P. Protter, Stochastic integration and differential equations. A new approach, Springer-Verlag, Berlin, 714, (1990).

[21] R. Savit, Nonlinearities and chaotic effects in options prices, J. Futures Mark., 9, 507-518 (1989).

[22] A. F. Soliman, A. M. .A. EL-ASYED, M. S. El-Azab, On The Numerical Solution of Partial integro-differential equations, Mathematical Sciences Letters, 1, 71-80 (2012).

[23] D. Sornette, Why Stock Markets Crash: Critical Events in Complex Financial Markets, Princeton University Press, Princeton, NJ, (2003).

[24] J. Tvedt, Valuation of european futures options in the bifex market, J. Futures Mark., 18, 167-175 (1998). 


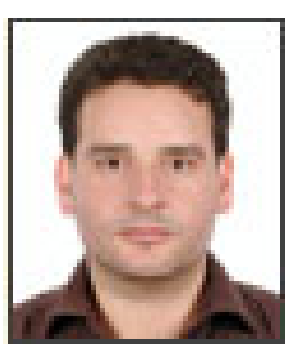

Youssef
El-Khatib
received the $\mathrm{PhD}$ degree
in Mathematics from the University of La Rochelle, France. His research interests are in the areas of stochastic calculus and applications to Finance including Malliavin (chaotic) calculus, pricing and hedging financial derivatives, computations of price sensitivities, stochastic volatility and jump-diffusion models. He has published research articles in international journals of Mathematics and Applied Finance. He is a referee for mathematical journals.

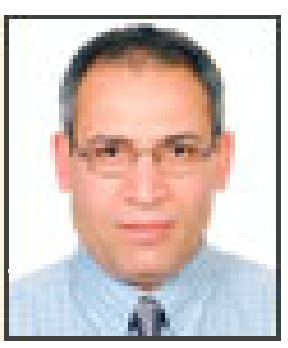

Mohamed Ali Hajji received the $\mathrm{PhD}$ degree in Applied Mathematics from Carleton University, Ottawa, Canada. His research interests center around scientific computing. His research works are in numerical solutions of DEs, wavelets and its applications. He published in many international journals. He is a referee for many international mathematical journals.

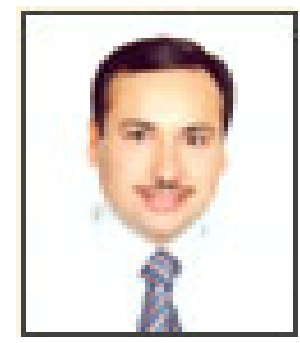

Mohammed Al-Refai. He received a $\mathrm{PhD}$ degree in Applied Mathematics from McGill University, Montreal, Quebec, Canada in 2000. His research interests are: numerical and analytical methods for ordinary and partial differential equations, maximum principle,

fractional calculus, non-local problems and financial Mathematics. He has about 20 publications in peer reviewed international journals. He acts as a referee for various international journals. 